\title{
Personalkosten planen und steuern
}

VON JOSEF BAUMÜLLER, THOMAS KLEIN UND SANDRA WILFINGER

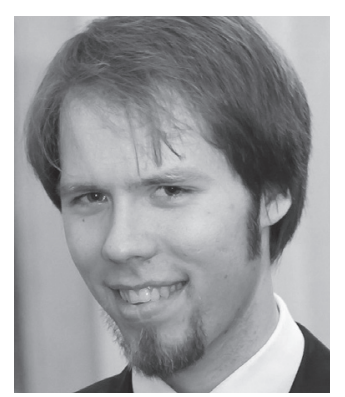

Josef Baumüller ist Universitätsassistent am Institut für Betriebswirtschaftslehre der Universität Wien. Zu seinen Forschungsschwerpunkten zählen rechtliche und wirtschaftliche Fragestellungen zu Non-ProfitOrganisationen.

josef.baumueller@univie.ac.at

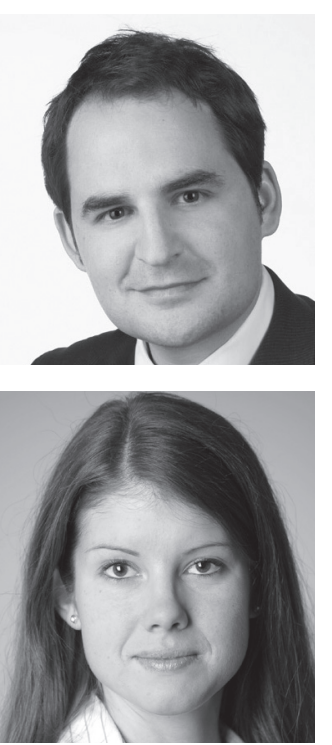

Thomas M. Klein und Sandra Wilfinger sind Unternehmensberater für Non-Profit-Organisationen und den öffentlichen Sektor bei Contrast Management-Consulting. Sie beraten Organisationen in den Fachbereichen Strategie, Organisation und Controlling. thomas.klein@contrast.at sandra.wilfinger@contrast.at

\author{
Der operativen Unternehmensplanung kommt \\ insbesondere in Form des jährlichen Budgets eine \\ hohe Bedeutung zu. In sozialen Organisationen \\ ist dabei insbesondere die richtige Planung der \\ Personalkosten eine stete Herausforderung, bei \\ der zahlreiche Aspekte zu berücksichtigen sind.
}

Non-Profit-Organisationen zählen in Deutschland zu den bundesweit größten Arbeitgebern. Dies liegt auch darin begründet, dass sie stark in Branchen vertreten sind, welche durch einen hohen Personaleinsatz gekennzeichnet sind: die Branchen der Sozialwirtschaft und des Gesundheitswesens. (1)

In Sozial- und Gesundheitsdiensten stellen die Personalkosten eine zentrale Größe dar, welche die finanzielle Stabilität der Organisation bestimmt. Erfahrungsgemäß fällt für dieses Kostensegment ein Anteil zwischen 70 und 90 Prozent der Gesamtkosten an. In Zeiten stagnierender oder reduzierter finanzieller Mittel seitens der Nutzer und der Fördergeber werden diese Personalkosten zu einer noch entscheidenderen Steuerungsgröße für das Controlling.

Die Planung der Personalkosten ist ein Teilaspekt der operativen Gesamtplanung und hat die Aufgabe, alle personalbezogenen Maßnahmen darzustellen, periodenbezogen zu bewerten und im Rahmen der Umsetzung zu überwachen. Die Gesamtplanung ist eine wichtige Basis für die Liquiditätsplanung und ermöglicht darüber hinaus die Ermittlung interner Kostensätze der Kostenträger. Die Plankosten können den geplanten Leistungsentgelten gegenübergestellt werden, um einen Kostendeckungsgrad zu ermitteln. Hieraus lässt sich bereits im Vorfeld der Handlungs- bedarf ableiten, etwa im Rahmen von Tarifverhandlungen. Im sozialen Sektor ist dieses Wissen jedoch oftmals noch nicht vorhanden, weil eine dafür notwendige Ermittlung von Kostensätzen für Einzelleistungen nicht erfolgt.

Die Kostenplanung ist der Planung der Leistungsmengen nachgelagert. (2) Da fixe Kosten als Kapazitäten gewisser Leistungsmengenintervalle verstanden werden, wird sich aus der Mengenplanung (auf Basis externer Vorgaben oder Marktentwicklungen) zunächst die Zahl und Struktur der Mitarbeiterinnen und Mitarbeiter ableiten lassen. Hohe Bedeutung hat für soziale Organisationen besonders die Frage nach den erforderlichen Mitarbeiterqualifikationen. Hier spielen auch personalstrategische Überlegungen eine Rolle, die sich häufig im Spannungsfeld »hohe Qualität, niedrige Kosten« bewegen. Zur Bewertung des resultierenden Mengengerüstes kann auf aktuelle Erfahrungswerte und (kollektiv-)vertragliche Entwicklungen abgestellt werden. Die Planung sollte direkt auf Ebene der Kostenstellen erfolgen.

Spätestens an diesem Punkt stellt sich die Frage nach der Plan-Auslastung, welche von den Mitarbeitenden zu erfüllen ist. Diese Kennzahl stellt im Controlling sozialer Organisationen eine der wichtigsten Steuergrößen dar. Hierfür ist zunächst auf zugängliche BranchenBenchmarks zurückzugreifen. Diesen 
ist die eigene erreichte Auslastung der Vorperioden gegenüberzustellen. Sich zeigende Abweichungen sind auf mögliche Gründe zu untersuchen, wobei gegebenenfalls entsprechende Maßnahmen abzuleiten sind. Aus der Berücksichtigung weiterer, für die kommende Planungsperiode erwarteter Sondereinflüsse ist letztlich die Planauslastung in den Qualifikationsgruppen festzulegen.

Hierbei werden sich die Kostenstrukturen »sprungfix" zu der Auslastung verhalten: In gewissen Intervallen wird eine Veränderung der Leistungsmengen zu einer Anpassung der (geplanten) Auslastung führen, jedoch zu keiner Anpassung der Kapazitäten selbst, also der fixen Personalkosten. Darüber hinaus wird sich entsprechender Anpassungsbedarf, beispielsweise durch einen Aufbau oder Abbau der Mitarbeitendenzahl, ergeben. Diese Analyse und Planung muss für einzelne Leistungsbereiche und Leistungsregionen untergliedert erfolgen, was idealerweise auch der Kostenstellenstruktur entspricht, anhand welcher die Planung erfolgt. In stark spezialisierten Abteilungsstrukturen kann zusätzlicher Ressourcenbedarf der einen Abteilung in der Regel nicht durch freie Kapazitäten einer anderen abgedeckt werden, $\mathrm{da}$ hierfür beispielsweise die erforderlichen Qualifikationen fehlen.

Bei größeren Anpassungen sind Vorlaufzeiten und damit verbundene Kosten zu berücksichtigen. So erfordert etwa ein geplanter Abbau der Zahl der Mitarbeitenden die Berücksichtigung von Kündigungsfristen, entsprechender Reduktionen der Leistungszeiten der betroffenen Mitarbeiterinnen und Mitarbeiter sowie gegebenenfalls weiterer Kosten, die in diesem Zusammenhang anfallen. Die Planung kann dabei nur grob anhand von Erfahrungswerten oder Schätzungen erfolgen. Die Literatur empfiehlt hierbei zunächst den Ansatz von periodisierten Durchschnittswerten als »laufende Vorsorge ", etwa in Form von Wagniskosten. Im Zusammenhang von voll geförderten Subventionsverträgen oder Leistungsentgeltvereinbarungen, die solche aperiodischen Schwankungen abdecken, sollten demgegenüber die tatsächlich im konkreten Planungszeitraum erwarteten Kosten angesetzt werden, allenfalls auch mittels Szenarien-Plänen. (3)

Da die meisten sozialen Organisationen von unterjährigen Schwankungen der geplanten Wertansätze betroffen sind, müssen diese in der Planung berücksichtigt werden. Lohnnebenkosten sind auf Jahresbasis als Zuschlagssätze zu den Grundgehältern zu ermitteln und gleich diesen linear über das Jahr $\mathrm{zu}$ verteilen. Andere typischerweise stark schwankende Kosten, wie etwa für Überstunden, können auf Basis der Vorjahreswerte geplant werden, so sie als Fixkosten klassifiziert wurden. Hinsichtlich Aus- und Weiterbildungskosten kann in einigen Fällen (z. B. Kongressteilnahmen) eine genaue unterjährige Planung erfolgen, in allen anderen scheint eine lineare Verteilung angemessen. Zuvor Gesagtes gilt sinngemäß auch für berücksichtigte Wagniskosten und Kosten ehrenamtlicher Mitarbeiterinnen und Mitarbeiter.

Ergebnis dieser Planung ist ein erwarteter Kostenverlauf für die folgende Periode. Für die unterjährige Kostenrechnung können hieraus StandardKostensätze für die interne Verrechnung ermittelt werden. Den Kostenverlauf gilt es im Berichtswesen zu überwachen, um gegebenenfalls Steuerungsbedarf festzustellen. Ein zentrales Instrument hierfür stellt der Soll-Ist-Vergleich dar. (4) Da jedoch die Effekte saisonaler Verschiebungen im unterjährigen Controlling zu berücksichtigen sind, kann nur die Erweiterung zur Plan-Wird-Rechnung die laufende Nachkalkulation der relevanten Leistungsentgelte gewährleisten. Die Planansätze des Budgets haben für die Budgetverantwortlichen verbindlichen Charakter in Form von Zielgrößen und Vergleichsmaßstäben. (5)

\section{Fazit und Ausblick}

Die Ausführungen unterstreichen die Bedeutung der Personalkostenplanung in sozialen Organisationen ebenso, wie sie deren Komplexität herausstreichen. Eine zu diesem Thema durchgeführte Studie des Österreichischen ControllerInstituts in Kooperation mit Contrast Management-Consulting unter österreichischen Non-Profit-Organisationen zeigte einen deutlichen Handlungsbedarf in der Praxis. (6) Erfreulicherweise lässt sich aber feststellen, dass sich immer mehr Organisationen der Wichtigkeit dieser Funktion bewusst werden und entsprechende Schritte unternehmen.

\section{Anmerkungen}

(1) Anheier et al. 2007, S. 25 ff.

(2) Egger/Winterheller (2007), S. 69.

(3) Seicht (2008), S. 71.

(4) Egger/Winterheller (2007), S. 122 ff.

(5) Eschenbach/Siller 2009, S. 234.

(6) Im Detail Horak/Baumüller (2009).

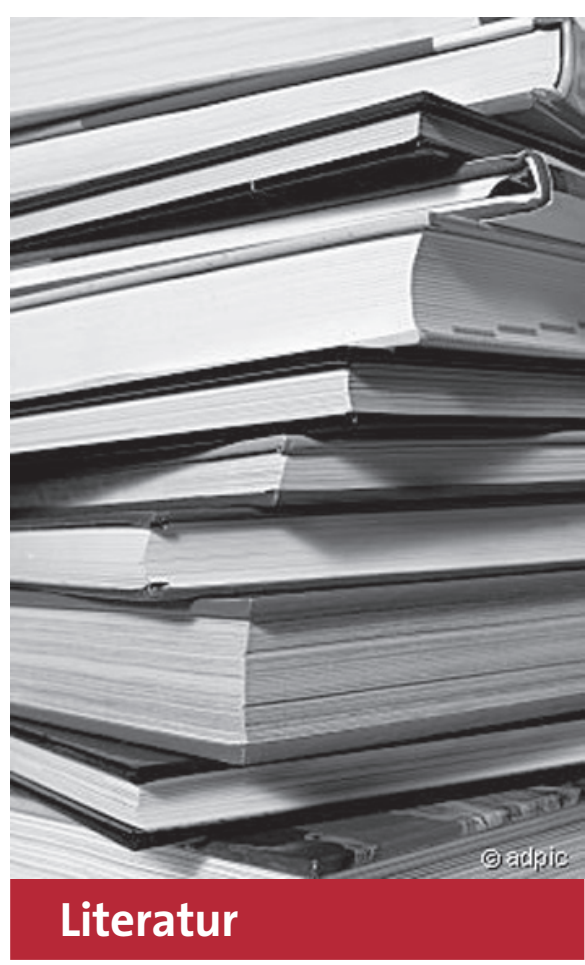

Anheier, Helmut K./Priller, Eckhard/Seibel, Wolfgang/Zimmer, Annette (2007): Der Nonprofit Sektor in Deutschland. In: Badelt, Christoph/Meyer, Michael/Simsa, Ruth (Hg.): Handbuch der Nonprofit Organisation, S. 17-39. 4. Auflage, Stuttgart.

Egger, Anton/Winterheller, Manfred (2007): Kurzfristige Unternehmensplanung. 14. Auflage, Wien.

Eschenbach, Rolf/Siller, Helmut (2009): Cont-

rolling professionell. Stuttgart.

Horak, Christian/Baumüller, Josef (2009): Steuerung in NPOs und der öffentlichen Verwaltung. Entwicklungsstand und Perspektiven. Eine empirische Studie des Österreichischen Controller-Instituts und Contrast ManagementConsulting, www.oeci.at.

Seicht, Gerhard (2008): Leitfaden Kosten- und Erfolgsrechnung. Wien. 